Maria Kaiafa-Gbandi

\title{
Europäisches Strafrecht - Die Perspektive des Grundrechtsschutzes nach dem Verfassungsentwurf für Europa
}

\section{Merkmale der Internationalisierung des Strafrechts in der EU}

Das Strafrecht wurde für die EU zu einem bedeutenden Rechtsgebiet, als diese grundsätzlich wirtschaftliche Staatenorganisation sich als solche stabilisiert hat und zu anderen Gebieten gemeinsamer Aktion tätig wurde. Der Wunsch für die möglichst größte Funktionalität des gemeinsamen Marktes und des freien Güter- und Personenverkehrs, wie auch der Wunsch für den Schutz ihrer wirtschaftlichen Interessen haben sie dazu geführt, das Strafrecht als das effektivste Mittel für die Verwirklichung ihrer Zwecke anzuwenden. ${ }^{1}$ Die Einflüsse der Globalisierung des Wirtschaftssystems sind außerdem bekannt für ihre Wirkung als zentrale Initiatorfaktoren der Internationalisierung des modernen Strafrechts. Sie bestimmen seine Merkmale mit, indem sie als Katalysator für möglichst gleiche Regelungen wirken, unter anderem über die Kontrolle der Einwanderungswellen von unerwünschten Ausländern, die Sicherung der Wettbewerbsregeln der verschiedenen Wirtschaftssystemen u.s.w. ${ }^{2}$ Dennoch, wenn man die hauptsächlichen internationalen Ebenen des modernen Strafrechts vergleicht, d.h. diese der UN, ${ }^{3}$ des Europarates und der EU, merkt man, dass obwohl die zwei ersten sich quantitativ auf mehreren Staaten öffnen, die intensivste Form der Internationalisierung des Strafrechts in der EU zu finden ist.

Die Merkmale dieser Internationalisierung möchte ich im folgenden kurz darstellen, damit man bessere Einsicht in ihre Folgen gewinnen kann.

Die Internationalisierung des Strafrechts im Rahmen der EU weist, meiner Meinung nach, drei grundlegende Merkmale auf. Es handelt sich nämlich um einen Prozess, der

1 Siehe dazu die wichtigen Änderungen des Amsterdamer-Vertags im Titel VI EUV und die Ergebnisse des Europäischen Rates von Tampere (Oktober 1999). Vgl. auch Monar, Die Entwicklung des »Raumes der Freiheit, der Sicherheit und des Rechts«. Perspektiven nach dem Vertrag von Amsterdam und dem Europäischen Rat von Tampere, Integration 2000, 18ff. und allgemeiner Kaiafa-Gbandi, The development towards harmonization within criminal law in the European Union-A citizen's perspective, European Journal of Crime, Criminal Law and Criminal Justice 2001, 239ff.

2 Siehe statt anderer Günther, Diethnis anteglimatiki politiki: I poria pros ena pagosmio dikaio asfalias, in Manoledakis/Prittwitz (Hrsg.), Diethnopiisi tou poinikou dikaiou, Athina-Thessaloniki, 2003, 29ff.

3 Über die Internationalisierungsmerkmale des Strafrechts, die bei der Errichtung des Internationalen Strafgerichtshofs zum Ausdruck gekommen sind, siehe Kaiafa-Gbandi, Die allgemeinen Grundsätze des Strafrechts im Statut des Internationalen Strafgerichtshofs: Auf dem Weg zu einem rechtsstaatlichen Strafrecht der Nationen?, in FS für H.-L. Schreiber, 200ff. und Manoledakis, I nea diethnopoiisi tou poinikou dikaiou kai o kindynos ypovathmisis tou nomikou mas politismou, Athina 2000, $18 \mathrm{ff}$. 
a) von dem tiefsten Eingriff in die Staatssouveränität begleitet wird,

b) inhaltlich gesehen ein Sicherheitsstrafrecht mit institutionell verallgemeinerten, d.h. undifferenzierten, Charakter im Bezug auf seinen Anwendungsbereich einführt und

c) die Grundrechte der Bürger in systematischer Form abbaut.

Im Einzelnen:

1. Der Internationalisierungsprozess im Rahmen der EU hat als Folge die bedeutendste Relativierung der Staatssouveränität, die sogar tiefer geht als diese, die sich mit der Gründung des Internationalen Strafgerichtshofs erreignet hat. ${ }^{4}$ Die EU tritt auf der Strafrechtsebene mit wachsender Intensität als Machtträger für die Bestimmung von Regelungen oder Aktionen auf und zwar, wie die Praxis zeigt, außer und über den ihr anerkannten Kompetenzen. Wenn man das Gebiet des Unionsrechts betrachtet, stellt man fest, dass in der Praxis der letzten Jahre die Einsetzung von Verträgen (Art. 34 Abs. 2d EUV) als Instrument der zwischenstaatlichen Kooperation im Bereich der polizeilichen und justiziellen Zusammenarbeit total marginalisiert worden ist. ${ }^{5}$ Und dies gerade weil die Ratifizierungsprozesse und die Möglichkeit von Vorbehaltserklärungen der Mitgliedsstaaten die von der EU erstrebten Ziele widersprechen. Mit anderen Worten befinden sich die Verträge als Rechtsinstrument viel näher zu den souveränen Staaten als zu der EU selbst und deshalb werden sie zur Ungebräuchlichkeit geführt. Andererseits erreicht die Ersetzung der Verträge durch die Rahmenbeschlüsse am Endeffekt das Durchsetzen der EU- Ziele viel besser. Denn die Rahmenbeschlüsse sind gleichfalls verbindlich hinsichtlich des zu erreichenden Ziels, wobei die für die Mitgliedsstaaten vorgesehene Möglichkeit über die Mittel und die Form zu entscheiden, in der Praxis durch den ausführlichen Regelungscharakter der Rahmenbeschlüsse zunichte gemacht wird. Mit anderen Worten stellt man hier die gleiche Haltung fest, die die EU bei den Richtlinien gezeigt hat, wo sie den Mitgliedstaaten

4 Vgl. aber Günther, Diethnis anteglimatiki politiki, o.a., 21. Die Parteien des Rom-Statuts haben mit einem internationalen Vertag dem IStGerichtshof Zuständigkeit für die strafrechtliche Verfolgung von bestimmten Delikte übertragen und ihre Souveränität aufgrund eines ganz bestimmten Textes eingeschränkt, welches dem Gerichtshof nur ergänzende Kompetenz anerkennt. Für künftige Änderungen des Vertrags muss eine neue Entscheidung der Parteien vorhanden sein. Dadurch ist ersichtlich, dass die Internationalisierung des Strafrechts, die mit dem Statut des IStGH zustande gekommen ist, sich innerhalb der klassischen Formen der Staaten-Kooperation bewegt, d. h. sie weist statischen Charakter auf, kann von sich selbst keine neue Regelungen produzieren und bezweifelt eigentlich nicht die Staatssouveränität. Natürlich wird hier ein rechtsprechendes Organ geschaffen, welches in konkreten Fällen die schwersten Delikte gegen die Menschheit auch gegen den Willen der betroffenen Staaten, die Vertragsparteien sind, bestrafen kann. Dennoch passiert dies im Einklang mit dem von ihnen auf abstrakter Ebene für solche Fälle ausgesprochenen Willen, wobei der Spielraum für die Bestrafung von Tätern aus Staaten, die nicht Vertragsparteien sind -und somit einer In-Fragestellung deren Souveränität- ist beschränkt bis ausgeschlossen. Eine solche Bestrafung setzt nämlich eine politische Entscheidung des UNSicherheitsrates voraus, bei dem die USA als ständiges Mitglied eine solche Möglichkeit voraussichtlich blockieren werden.

5 Man braucht dazu einfach einen Blick auf die Rechtsinstrumente der letzten Jahre zu werfen, die im Gebiet des VI Titels eingesetzt worden sind. Dabei findet man keine Verträge des Art. 34 Abs. 2 d EUV mehr. 
eigentlich keinen Spielraum für wesentliche Entscheidungen übriglässt. ${ }^{6}$ Diese Praxis verstößt sicherlich gegen Grundprinzipien der Rolleneinteilung zwischen den Mitgliedstaaten und der Union (Subsidiaridätsprinzip), wurde aber trotzdem im Falle der Richtlinien vom Gerichtshof der Europäischen Gemeinschaften nicht beanstandet. ${ }^{7}$

Somit wird klar, dass auf der Ebene der intergouvernementalen Kooperation auch, auf der man sagen würde, dass die Staatssouveränität vielmehr bestätigt als bestritten wird, die Verflechtung der Mitgliedstaaten und der Union auf eine in der Tat Oberhand der Letzteren hinausläuft. Nicht nur weil die Union zu einer Grenzüberschreitung der ihr zuerkannten Kompetenzen tendiert, ${ }^{8}$ aber auch weil die Mitgliedstaaten ihre Rolle nicht wahrnehmen, damit sie solche Überschreitungen abwenden. Um ein vollständiges Bild zu haben, lohnt es sich vor Augen zu halten, dass die Völker Europas auf dem Bereich der im dritten Pfeiler entstehenden strafrechtlichen Materie kein Mitbestimmungsrecht haben, da das Europäische Parlament hier nur eine Zustimmungsbefugnis hat (Art. 39 EUV). Somit wird auf der Ebene des Unionsrechts nicht nur der Begriff der Staatssouveränität entscheidend relativiert, sondern wird auch die allmählich aufkommende Souveränität der Union von ihrer Zurückführung zum Volk als letztendlichen Machtträger losgelöst.

Die Lage unterscheidet sich nicht viel auch auf der Ebene des Gemeinschaftsrechts. Ich errinere hier an den Vorschlag der Kommission für eine Richtlinie bezüglich des strafrechtlichen Schutzes der wirtschaftlichen Interessen der EU im Rahmen des geltenden Status der Verträge, ${ }^{9}$ obwohl der Gemeinschaft bis heute keine strafrechtliche Kompetenz zuerkannt wurde.

Man merkt dadurch, dass die EU eine sich dynamisch entwickelnde Organisation darstellt, die nicht selten weit und über der ihr zuerkannten Macht handelt und eine ungeheuere Menge strafrechtlicher Materie produziert, die sie mit mittelbaren oder unmittelbaren Mechanismen in den Mitgliedstaaten durchzusetzen versucht. Dabei überflügelt sie nicht selten die Beteiligung der Mitgliedstaaten und zwar ohne an den Völker Europas als souveränen Gesetzgeber durch das Europäische Parlament etwa Rechenschaft zu geben. Hier ist die Produzierung vom Strafrecht mit einer europäischen Exekutive sehr eng verbunden, deren Zwecke sie auch verfolgt. ${ }^{10}$ Somit

6 Siehe Kaiafa-Gbandi, To poiniko dikaio stin Europaiki Enosi, Simantikes opsis kai prooptikes exelixis, Athina-Thessaloniki, 2003, 283 und Fn. 12 und Musil, Umfang und Grenzen europäischer Rechtssetzungsbefugnisse im Bereich des Strafrechts nach dem Vertrag von Amsterdam, NStZ 2000, 70.

7 Diesbezüglich Sachpekidou, in Stangou-Sachpekidou, Dikaio ton Europaikon Koinotiton kai tis Europaikis Enosis, Thessaloniki 2000, 209.

8 Beispiele dazu siehe bei Kaiafa-Gbandi, To poiniko dikaio stin Europaiki Enosi, (o.a.), 198 ff, $251 \mathrm{ff}$ und Tsolka, Europaiko entalma syllipsis, Poinika Chronika 2002, 107.

9 Siehe COM 2001, 272 und dazu Kaiafa-Gbandi, Europaiko poiniko dikaio, (o.a.), 277ff. Vgl. kritisch über das Problem der Strafrechtsetzungskompetenz der Gemeinschaft vom Aspekt der demokratischen Legitimation Prittwitz, Nachgeholte Prolegomena zu einem künftigen Corpus Juris Criminalis für Europa, ZStW 2001,790-791.

10 Vgl. P. A. Albrecht, Prostasia tis eleuthrias: Kathikon tis europaikis exelixis tou poinikou dikaiou, in Manoledakis/Prittwitz, Diethnopoisi tou poinikou dikaiou, Athina-Thessaloniki 2003, 210ff., Lüderssen, Europaiki enopoissi tou poinikou dikaiou kai kyvernitiki dikaiothesia, in Manoledakis/Prittwitz, Diethnopoissi tou poinikou dikaiou, Athina-Thessaloniki 2003, 171ff. 
könnte man sagen, dass die EU im Gebiet des Strafrechts allmählich ihre eigene Souveränität herstellt, indem sie die Souveränität der Mitgliedstaaten abbaut, ohne dass sie in diesem Verfahren die Zurückführung dieser Souveränität an der Gesamtheit der Völker Europas durch eine Selbstbegrenzung durch das Recht sicherzustellen. Die Erklärung dieses Phänomens liegt in mehreren Gründen, die hier nicht erläutert werden können. Für ein rechtsstaatliches europäisches Strafrecht ist das aber ein unannehmbares Phänomen. Denn für die Bestimmung von Strafe für die Bürger als den tiefsten Eingriff in ihre Freiheit ist, wie bekannt, nur ein Organ legitimiert, welches das Volk als Machtträger in der repräsentativsten Art und Weise vertritt. Und dies ist nur das Parlament. ${ }^{11}$ Deshalb sollte, meiner Meinung nach, die obenbeschriebene Lage geändert werden und zwar mit Anerkennung voller und ausschließlicher Zuständigkeit des Europäischen Parlaments für die Verabschiedung strafrechtlicher Materie, ${ }^{12}$ was leider auch mit dem Verfassungsentwurf für Europa nicht passiert ist. ${ }^{13}$

2. Das zweite Merkmal der Internationalisierung des Strafrechts im Rahmen der EU hat mit dem Inhalt der produzierten Regelungen und deren Bezugsobjekt zu tun. Hier, wie bei den anderen Internationalisierungsebenen des Strafrechts auch, wird heute hauptsächlich ein Sicherheitsstrafrecht produziert. ${ }^{14}$ Dennoch liegt die Besonderheit mit der EU darin, dass innerhalb ihrer Grenzen zum ersten Mal auf internationaler Ebene eine ausdrückliche institutionelle Anerkennung der Umorientierung des Strafrechts zur Sicherheit festzustellen ist und zwar in den Artikeln 2 und 29 EUV, ${ }^{15}$ wo als zentrales Ziel für die Union die Gewährleistung eines hohen Maßes an Sicherheit für die Bürger dargestellt wird.

Das bedeutende Element dabei ist, dass in dieser Weise ein festes und fortdauerndes Sicherheitsziel gesetzt wird, dem jede strafrechtliche Materie im Rahmen der EU dienen soll. Das Sicherheitsziel ist mit anderen Worten hier weder gelegentlich noch eines »einmaligen Gebrauchs«, wie der Fall bei den zwischenstaatlichen Kooperationen durch Verträge normalerweise ist, sondern es hat allgemeine Form erhalten. Und das schlimmste ist, dass es sich nicht mal ausschließlich auf das Gebiet der organisierten Kriminalität oder des Terrorismus beschränkt, da die Regelung von Art. 29 EUV über die Erreichung dieses Ziels »durch die Verhütung und Bekämpfung der organisierten oder nicht organisierten Kriminalität« spricht. Die gemeinsame Behandlung der organisierten mit der nicht organisierten Kriminalität im Rahmen eines allgemeinen Sicherheitsziels ist aber evident, dass gegen die Letztere funktioniert, da die außerordentlichen Maßnahmen, die normalerweise für die organisierte Kriminalität vorgenommen werden, bei der nicht organisierten vielmehr nicht gerechtfertigt werden können. Und diese ist gerade die Gefahr, die der Amsterdamer Vertrag mit Art. 29

11 Siehe Lüderssen, o.a., 193-194.

12 Siehe Kaiafa-Gbandi, To poiniko dikaio stin Europaiki Enossi, (o.a.), 299 und Pradel, Avis sur la sanction des violations du droit communautaire par le droit pénal, (in Erscheinung, Elliniki Epitheorisi Europaikou Dikaiou, 2004, Bd. 1). Vgl. dazu Lüderssen, o.a., 197.

13 Siehe weiter unten, S. 21-22.

14 Siehe diesbezüglich $P$-A Albrecht, Prostasia tis eleutherias: Kathikon tis europaikis exelixis tou poinikou dikaiou, (o.a.), 200ff. und Günther, Diethnis anteglimatiki politiki (o.a.), 29ff.

15 Vgl. Günther, o.a., 25. 
EUV institutionalisiert hat, nämlich das verallgemeinerte und undifferenzierte Überwiegen der Sicherheit im Strafrecht oder anders formuliert die institutionelle Anerkennung der dynamischen Reaktionen, die normalerweise die gesetzten Grenzen oder das Verhältnismäßigkeitsprinzip nicht beachten.

3. Das dritte wesentliche Merkmal hat mit dem Grundrechtschutz der Bürger zu tun. Im Konkreten, wobei der Rückgang der Staatssouveränität gegenüber der EU als Träger bestimmter Kompetenzen auch im Strafrechtsgebiet aufgrund der jeweiligen Regelungen des dritten Pfeilers anscheinend für den besseren Schutz der Rechtsgüter vorgenommen wurde, und als solcher eigentlich einen allgemeineren positiven Einfluss haben sollte, wurde in der Praxis von einem Abbau des Grundrechtsschutzes begleitet, der darauffolgend den Druck für seine Weiterbeförderung auf die Mitgliedsstaaten überführt hat. Andererseits, obwohl jetzt die Grundrechte der Bürger auch vom Handeln europäischer Organe angegriffen werden können, ist deren institutioneller Schutz auf Unionsebene nicht sichergestellt worden.

Die festzustellende Besonderheit liegt also hier darin, dass die den Grundrechtsschutz abbauende Regelungen der EU von Organen einer Exekutive im Grunde genommen produziert werden, was eigentlich auf ihre engere Gebundenheit an eine angriffsneigende und demokratisch nicht kontrollierte Sicherheitspolitik hinweist, wobei die Mitgliedstaaten, andererseits, für die Überführung dieser Regelungen in ihr nationales Recht unmittelbarer und effektiver als im Rahmen der klassischen internationalen Verträgen verpflichtet sind. Wenn man dazu an den Mangel eines eigenen institutionellen Schutzmechanismus der EU für die Grundrechte der Bürger und ihre Ablehnung in die EMRK beizutreten denkt, wie auch an die Tatsache, dass ihre Regelungen im Bereich der polizeilichen und justiziellen Zusammenarbeit in Strafsachen sich mit schwindelerregender Geschwindigkeit vermehren, dann hat man ein Bild vom Ausmaß des Problems, welches ich vorhin »verallgemeinertes Abbauprozess des Grundrechtschutzes« genannt habe.

\section{Der Abbau des Grundrechtsschutzes}

Diesen Abbauprozess, den ich als die wichtigste Besonderheit der Europäisierung des Strafrechts halte, möchte ich an drei ausgewählten Beispielen zeigen. Das erste Beispiel kommt aus dem Gebiet der Begriffbestimmung des organisierten Verbrechens. Das zweite Beispiel wiedergibt das Verhältnis der polizeilichen und justiziellen Macht in der EU und dessen Folgen und das dritte Beispiel beschäftigt sich mit dem ersten Auftritt der EU im internationalen Raum in Fragen der Rechtshilfe in Strafsachen mit der Unterzeichnung der relevanten Abkommen mit den Vereinigten Staaten Amerikas.

1. Im Rahmen der Auffassung der EU über die Begriffsbestimmung des organisierten Verbrechens hat man die Möglichkeit genau mitzuverfolgen, wie das Strafrecht allmählich vom Recht der Tat zum Recht der Gesinnung umgewandelt wird und seine Garantiefunktion für den Bürger verliert.

Die EU hat schon im Dezember 1998 - im Rahmen des vor Amsterdam geltenden Status der Verträge- eine Gemeinsame Maßnahme erlassen, mit der sie versucht hat, ihre Auffassung über die Strafbarkeit der Beteiligung an einer kriminellen Vereini- 
gung als hauptsächliches Kontrollinstrument des organisierten Verbrechens in den Mitgliedstaaten durchzusetzen. ${ }^{16}$ Trotz der Tatsache, dass nicht wenige Mitgliedstaaten dem Inhalt dieser Maßnahme nicht Folge geleistet haben,${ }^{17}$ hat die EU sie dazu aufgefordert, für die Kompatibilität der Regelungen des damals noch als Entwurf diskutierten Vertrags der Vereinten Nationen über die Bekämpfung des transnationalen organisierten Verbrechens mit ihrer Gemeinsamen Maßnahme zu sorgen, ${ }^{18}$ wobei sie auch nach dem In-Krafttreten des Amsterdamer-Vertrags fortführt, sich auf diese Maßnahme ausdrücklich zu berufen, sowohl um auf ihrem Inhalt die Zuständigkeiten von neuen Organen, wie Eurojust, zu basieren, ${ }^{19}$ wie auch um den Mitgliedstaaten Pflichten für strengere Bestrafung derjenigen Delikte aufzuerlegen, die normalerweise in organisierter Form begangen werden. ${ }^{20}$ Mit anderen Worten, bietet die Gemeinsame Maßnahme von 1998 über die Strafbarkeit der Beteiligung an einer kriminellen Vereinigung den Schlüssel zum Verständnis der Union über das organisierte Verbrechen und deshalb ist diese Maßnahme von besonderer Bedeutung. ${ }^{21}$

Wenn man nun die einzelnen Regelungen dieser Maßnahme vom 1998 durchliest, merkt man, dass es ihr gelungen ist, den Begriff der Beteiligung an einer kriminellen Vereinigung in bedeutender Weise zu erweitern, im Vergleich zu dem, was die Union selbst für das organisierte Verbrechen bis dahin vertreten hat. ${ }^{22}$ Im Konkreten erlaubt

16 98/733/JI, L 351/1-2 vom 29.12.1998.

17 Dazu insbesondere Mitsilegas, Defining organised crime in the European Union: the limits of European Criminal Law in the area of »freedom, security and justice«, ELReview 2001, 574 und Fn. 35.

18 Siehe die gemeinsame Position des Rates vom 29.3.1999 (1999/235/JI, L087 vom 31.3.1999, S. 1-2) und insbesondere Art 2.

19 Siehe Art. 4 des Ratsbeschlusses vom 28.2.2002 über die Errichtung von Eurojust (2002/ 187/JI, L 63 vom 6.3.2002, S. 1-13).

20 Siehe Art. 3 Abs. 2d des Rahmenbeschlusses über die Bekämpfung des Menschenhandels (2002/629/JI, L 203 vom 1.8.2002, S. 1-4).

21 Im Bezug auf diese Gemeinsame Maßnahme sind zwei Sachen wichtig: Erstens, dass der Gerichtshof der Europäischen Gemeinschaften keine Zuständigkeit hat, über die Gültigkeit und die Auslegung solcher Maßnahmen zu entscheiden, da sie als Rechtsinstrumente des vor Amsterdam-Status der Verträge im Artikel 35 EUV nicht erwähnt werden. Zweitens, dass die Frage der Weitergeltung solcher Maßnahmen, obwohl sie nach dem AmsterdamerVertrag von den Rahmenbeschlüssen ersetzt worden sind, positiv geantwortet werden soll, denn das ist vom Prinzip der Weitergeltung des Rechts zu entnehmen (siehe dazu auch Mitsilegas, ELReview 2001, 579). Das macht verständlich, warum es angebracht erscheint, diese Maßnahme mit einem modernen Rechtsinstrument zu ersetzen, denn somit wird es zumindest der Zuständigkeit des Gerichtshofs unterstellt.

22 Vgl. dazu den Kriterienkatalog des Europäischen Rates, anhand dessen die Mitgliedstaaten seit 1994 ihre jährlichen Berichte zum Stand der organisierten Kriminalität innerhalb ihrer Grenzen erstellen und nach dem die folgenden 4 Merkmale unbedingt notwendig sind, damit man über organisierte Kriminalität spricht: a) eine Organisation bestehend aus mehr als zwei Personen, b) Zusammenarbeit ihrer Mitglieder für eine bedeutende oder unbestimmte Zeit, c) Verdacht der Begehung von schweren Delikten und d) Hauptzweck der Erlangung geldwerter Vorteile oder Macht (Bleeker, The criminal justice system facing the challenge of organised crime, International Review of Penal Law 1999, 648-649, Bruggeman, The fight against organized crime: possibilities, problems and opportunities with a special focus on the EU, in H.-J. Albrecht/ C. Finjaut, The Containment of Transnational Organized Crime, 2002, 68-69). 
die Beschreibung der kriminellen Vereinigung darunter auch Vereinigungen zu subsumieren, die keine Dynamik einer bedeutenden und selbstständigen Gefährdung der öffentlichen Ordnung aufweisen, weil weder die Widmung der Tätigkeit solcher Vereinigungen an schweren Straftaten ${ }^{23}$ noch der Zweck der Beschaffung eines wirtschaftlichen oder eines anderen materiellen Vorteils gefordert wird. Andererseits wird eine speziell bestimmte Organisationsform des Zusammenschlusses der zumindest drei Personen nicht vorausgesetzt, wobei die Beteiligung auch, also die entscheidende Straftat, um die es hier geht, keine Beteiligung an der Vereinigung selbst zu sein braucht, ${ }^{24}$ sondern eine Beteiligung an verschiedenen strafbaren Tätigkeiten, im Vorbereitungsstadium der Delikte, also ein Verhalten von dem der spezielle Unwert einer Gefährdung der öffentlichen Ordnung, mit der das organisierte Verbrechen gebunden wird, sich eigentlich nicht ergeben kann. ${ }^{25}$ Vielmehr kann dieser Unwert sich nicht von der weiteren Öffnung der Gemeinsamen Maßnahme vom 1998 zur Strafbarkeit der Beteiligung von außenstehenden Personen ergeben, für die objektiv ausreichend ist, dass sie sich an den sonstigen, also den nicht strafbaren, Tätigkeiten der Vereinigung beteiligen ohne jedwelche weitere Voraussetzung, also ohne eine Verbindung dieser Beteiligung mit Taten des Aufbaus, der Existenz oder der operationellen Funktion der Vereinigung. ${ }^{26}$

23 Die Voraussetzung der Begehung von Straftaten, die mit einer Freiheitsstrafe von einem bestimmten Mindest-Höchstmaß bestraft werden (siehe so Art. 1 der Gemeinsamen Maßnahme vom 1998), reicht wohl nicht aus, um die Identifizierung der begangenen Taten als schwere Delikte zu wiedergeben, wenn man über die Strafbarkeit der Beteiligung an einer kriminellen Vereinigung im internationalen Rahmen spricht und das schon wegen der Unterschiede bei den Strafgrossen in den verschiedenen Staaten. (Vgl. diesbezüglich auch die Kritik von Militello, Participation in an organized criminal group as international offence, in Militello/Huber (eds.), Towards a European Criminal Law against Organized Crime, Freiburg .i. Br. 2001, 102). Deshalb hat es immerhin einen Sinn für die Begriffsbestimmung einer kriminellen Vereinigung, zusätzlich die Plannung der Begehung schwerer Straftaten von ihr ausdrücklich zu fordern.

24 Wenn die Gemeinsame Maßnahme die Beteiligung der Person als Mitglied der kriminellen Vereinigung voraussetzen würde, dann würde der besondere Unwert der erwünschten Strafbarkeit aus der Tat der jeweiligen Person hervorgehen. Denn die Mitgliedschaft an einer solchen Vereinigung mit einer gewissen Dauer und die Übernahme einer Rolle darin könnten den individuellen Beitrag der Person durch ihr eigenes Verhalten zum speziellen Unwert einer wesentlichen Gefährdung der öffentlichen Ordnung von der Funktion einer solchen Vereinigung zum Ausdruck bringen.

25 Über diesen speziellen Unwert siehe insbesondere Manoledakis, Asfalia kai eleutheria, Thessaloniki, 2002, 104-106, Livos, Organisiertes Verbrechen: Ennia kai dikonomikoi tropoi antimetopisis tou, in Praktika Z' Panelliniou Synedriou EEPD, Athina 2000, 48-51 und Kaiafa-Gbandi, To poiniko dikaio stin Europaiki Enosi, 154ff.

26 Für die Notwendigkeit ähnlicher Voraussetzungen siehe insbesondere Militello, Towards a European Criminal Law against organized crime-Research in brief, Freiburg i.Br. 2001, 8. Vgl. dazu die fehlende Klarstellung der Grenzen zwischen einem legalen Dienstangebot und der strafbaren Unterstützung einer kriminellen Vereinigung von außenstehenden Personen im Rahmen einer von ihnen legalen Ausübung ihres Berufs. Über diese Problematik siehe schon Weigend, The criminal justice system facing the challenge of organized crime, Revue internationale de droit pénal 1997, 530-531 und ausführlicher über dasselbe Problem mit der Gemeinsamen Maßnahme vom 1998 Kaiafa-Gbandi, To poiniko dikaio stin Europaiki Enossi (o.a.), 161ff. 
Sicherstellung von der Gefahr einer übermäßigen Erweiterung der Strafbarkeit können außerdem auch nicht die subjektiven Elemente anbieten, die die obenbeschriebenen Verhalten abdecken sollen. Denn hier mag die geforderte Kenntnis des Ziels der allgemeinen strafbaren Tätigkeit der Vereinigung oder deren Absicht, die betreffenden Straftaten zu begehen, theoretisch die Strafbarkeit begrenzen, aber sie setzt selbst die subjektive Abdeckung von subjektiven Elementen anderer Personen (!) - und zwar mehrerer - voraus. Wie schwierig und unsicher ein solches Schema ${ }^{27}$ bei der Annäherung des Vorsatzes $^{28}$ und dementsprechend funktionell die Strafbarkeit begünstigend wird, braucht nicht weiterhin ausgeführt zu werden.

Der Rückgang, den die Freiheit zugunsten der gezielten Sicherheit augrund solcher Regelungen erfährt, ist evident und zwar nicht nur wegen der bei der Gemeinsamen Maßnahme offen gebliebenen Möglichkeit für die Orientierung der Strafbarkeit an die angelsächsische Institution der »conspiracy $« .{ }^{29}$ Denn auch die Verbindung der Strafbarkeit mit Tätigkeiten von kriminellen Vereinigungen kann hier Taten betreffen, die von sich selbst keinen speziellen, für das organisierte Verbrechen charakteristischen, Angriff der öffentlichen Ordnung aufweisen. Somit tritt aber die Tat als Grundträger des Unwerts notwendigerweise zurück und an ihrer Stelle wird der Vorsatz berufen, um den strafbaren Unwert hauptsächlich zum Ausdruck zu bringen. Oder anders ausgedrückt: die für die Strafbarkeit notwendige Tat, wird somit normalerweise, aufgrund des breiten Spektrums der Verhalten, die hier relevant werden, sehr einfach zu finden sein, wobei die Zurechnung des Vorsatzes, der hier den Unwert konkretisiert, nicht mehr aufgrund der begangenen Tat stattfinden wird, denn die Tat selbst kann diesen Unwert nicht mehr ausdrücken. Somit wird aber das für den Rechtsstaat grundlegende Verhältnis von objektiven und subjektiven Elementen des Verbrechens, welches den strafbaren Unwert an erster Stelle bei der Tat einer Person aufsucht, total umgekehrt ${ }^{30}$

27 Siehe dazu auch die Kritik von Verbruggen, On containing organized crime using »container offences «, in Albrecht H.-J./ Fijnaut C. (eds.), The Containment of Transnational Organized Crime, Freiburg i.Br. 2002, 131.

28 Hier sollte man an die Dispositionsbegriffe denken, zu denen der Vorsatz auch unterzuordnen ist und an deren Annäherung durch Indikatoren, die zumindest beobachtbar sein sollen (diesbezüglich Hassemer, Die Freiwilligkeit beim Rucktritt vom Versuch, in Lüderssen/ Sack (Hrsg.), Vom Nutzen und Nachteil der Sozialwissenschaften für das Strafrecht, Tb. 1, 243ff., Kaiafa-Gbandi, Exoteriki kai esoteriki amelia sto poiniko dikaio, Thessaloniki 1994, 172ff., Mylonopoulos, Diathetikes ennies kai poiniko dikaio, Yperaspissi 1993, 247ff.) .

29 So Artikel 2 Abs. 1 b der Gemeinsamen Maßnahme von 1998 (98/733/JI), der den Mitgliedstaaten die Möglichkeit offen läßt, entweder die Beteiligung an einer kriminellen Vereinigung oder die Vereinbarung mit einer anderen Person über die Begehung einer Straftat oder beides unter Strafe zu stellen.

30 Diese Umkehrung und andere Ausnahmen vom rechtsstaatlichen Strafrecht akzeptiert aber Jakobs (Das Selbstverständnis der Strafrechtswissenschaft vor den Herausforderungen der Gegenwart, Eser/Hassemer/Burckhardt (Hrsg), Die deutsche Strafrechtswissenschaft vor der Jahrtausendwende, München 2000, 51-54) und stellt sogar eine theoretische Grundlage für »ein Feindstrafrecht « bei der Bekämpfung der organisierten Kriminalität vor. Für eine Auseinandersetzung mit seinen Argumenten in der gr. Literatur siehe Kaiafa-Gbandi, To poiniko dikaio stin Europaiki Enosi, 167-168, Fn. 88. 
mit schweren Folgen auch für den prozessualen Raum der Ermittlungen. Die verdeckte Ermittlungen bekommen nämlich gerade dort herrschende Rolle, wo eine Tat aufzudecken ist, deren Unwert sich hauptsächlich auf subjektive Elemente basiert. ${ }^{31}$

Ähnliche Probleme schafft auch der Rahmenbeschluss über die Terrorismusbekämpfung. ${ }^{32}$

Wie man von meinem ersten Beispiel feststellen kann, fängt der Abbau der Grundrechte des Bürgers mit der rechtsstaatlich besonders problematischen Abgrenzung der Strafbarkeit im Bezug auf ihre niedrigsten Grenzen an, die die EU den Mitgliedstaaten vorzuschreiben versucht. ${ }^{33}{ }^{34}$ Denn dieser Druck für eine Neubestimmung der untersten Strafbarkeitsgrenzen kann bedeutende Umstellungen zu einem Gesinnungsstrafrecht mit sich bringen.

2. Das zweite Beispiel betrifft nicht mehr das Gebiet der Strafbarkeitsbestimmung, sondern dieses der Betätigung unionseigener Organe für die Verhütung und Bekämpfung von Straftaten. Im Rahmen der Union haben wir hier auch eine Besonderheit, die vom Anfang an klar sein sollte. Gerade weil sie in diesem Gebiet über unmittelbare Kompetenz für die Strafverfolgung nicht verfügt, basierte sich ihre Aktivität, geschickter Weise, auf transnational gebaute Organe, zu denen sie, dem äußeren Schein nach zumindest, Koordinierungskompetenzen gegeben hat. Dadurch hat sie versucht sowohl den Einwand der ihr fehlenden strafrechtlichen Zuständigkeit zu entgehen, wie auch diesen der Notwendigkeit der Schaffung eines übernationalen Kontrollmechanismus für den Grundrechtsschutz der Bürger. Gleichzeitig ist es wichtig vor Augen zu halten, dass die polizeiliche und justizielle Zusammenarbeit für die EU den wichtigsten Faktor ihrer Intervention im Bereich des Strafrechts darstellt, d.h. viel wichtigeren als eine eventuelle Harmonisierung der jeweiligen nationalen Rechtsordnungen. Denn die Letztere ist schwierig und zeitaufwendig, aber gleichzeitig auch leicht umgehbar, wenn man denkt, dass schließlich viele von den Rechtsunterschieden der Mitgliedsstaaten beseitigt oder unbedeutend werden können, durch eine justizielle Zusammenarbeit auf der Basis des Prinzips »der gemeinsamen Anerkennung von strafrechtlichen Entscheidungen«. Die EU sorgt sogar dafür, dass diese Zusammenarbeit den möglichst weiten Wirkungsraum bekommt, hauptsächlich durch eine weite Öffnung der Gerichtsbarkeit der Mitgliedstaaten zumindest bei der Bekämpfung der organisierten Kriminalität ${ }^{35}$ und des Terrorismus. ${ }^{36}$

31 Vgl. Verbruggen, On Containing organized crime using »container offences « (o.a.), 131.

32 Vgl. Art. 2 Abs. 2 des Rahmenbeschlusses vom 13.6.2002 (2002/475/JI), L 164 vom 22.6.2002, S. 3-7.

33 Siehe Art. 5 Abs. 2 der Gemeinsamen Maßnahme vom 1998 (98/733/JI), der vorsieht, dass diese Maßnahme die Mitgliedstaaten nicht hindert, Verhalten bezüglich einer kriminellen Vereinigung unter Strafe zu stellen, das über die von ihr beschriebenen Verhaltensweisen hinausgeht.

34 Zur Zeit zeigt die EU im Bezug auf das organisierte Verbrechen zumindest eine Wartehaltung, die mit den zu erwartenden Ergebnissen der Unterzeichnung des UN-Vertrags über die Bekämpfung des transnationalen organisierten Verbrechens von den Mitgliedstaaten zusammenhängt. Das heißt eine Neubetätigung der EU in diesem Gebiet ist gar nicht auszuschliessen. Vgl. Betti, The EU and the UN Convention against Transnational Organised Crime (Working Paper European Parliament), Civil Liberties Series, LIEBE 116 EN, iii-iv, $103 f f$. 
Das Beispiel, das ich hier ausführe, versucht den Abbau der Grundrechte auf diesem Gebiet zu zeigen, indem es bis zum institutionellen Verhältnis von Europol und Eurojust reicht, denn von der Letzteren hat man erwartet, dass sie zumindest als Gegenpol zu der polizeilichen Betätigung der Union wirken würde. ${ }^{37}$

Wie bekannt, liegt das Hauptgewicht der Zuständigkeit von Europol heute noch an der Weitergabe von Informationen zu den zuständigen Behörden der Mitgliedstaaten und an der Datenanalyse für die transnationale Kriminalität eines sehr breiten Spektrums von Straftaten. ${ }^{38}$ Dennoch sind auch die Defizite des Rechtsschutzes bekannt, der den Bürgern im Bezug auf das Recht ihrer informationellen Selbstbestimmung gegenüber der Funktion von Europol angeboten wird ${ }^{39}$ und zwar insbesondere wegen der Immunitäten von Europol, ${ }^{40}$ die trotz der Änderung ihres Gründungsvertrags für alle andere Fälle außer ihrer Teilnahme an gemeinsamen Ermittlungsteams beibehalten wurden. ${ }^{41}$ Die Regelung über die Aufhebung der Immunitäten von Europol ist absolut unvereinbar mit den Grundsätzen unserer europäischen Zivilisation, da sie immer von Europolsdirektor und aufgrund ihrer Interessen entschieden wird, wobei diese Entscheidung nur vom Rat kontrolliert werden kann, also von einem Organ der Exekutive eigentlich. ${ }^{42}$ Diese Immunitäten sind in der Lage den Schutz der Bürger bei den nationalen Gerichten zu annullieren, wobei der Schutz, den die sogenannte Gemeinsame Kontrollinstanz anbietet, nicht zufriedenstellend ist, da diese Instanz

35 Siehe Art. 4 der Gemeinsamen Maßnahme vom 21.12.1998 (98/733/JI), L351 vom 29.12.98, S. 2.

36 Siehe Art. 9 des Rahmenbeschlusses vom 13.6.2002 (2002/475/JI), L 164 vom 22.6.2002, S. 8.

37 Dazu beispielsweise Berthelet-Chevallier/Govers, Quelle Relation entre Europol et Eurojust? Rapport d' egalité ou rapport d' autorité?, Revue du Marché commun et de l' Union européenne 2001, 474, Schomburg, Are we on the road to a European Law-Enforcement Area? International Cooperation in Criminal Matters. What place for justice?, European Journal of Crime, Criminal law and Criminal Justice 2000, 59 aber auch COM (2000) 746 final, Brussels 22.11.2000.

38 Siehe Art. 3 des Übereinkommens über die Errichtung eines Europäischen Polizeiamts (C 316 vom 27.11.1995, S. 2) und seine Änderungen durch den Rechtsakt des Rates vom 28.11.2002 ( C 312 vom 16.12.2002, S. 1) und den Rechtsakt des Rates vom 27.11.2003 (C 2 vom 6.1.2004, S. 1) wie auch von Bubnoff, Institutionelle Kriminalitätsbekämpfung in der EU- Schritte auf dem Weg zu einem europäischen Ermittlungs- und Strafverfolgungsraum, ZeuS 2002, 192, Harding, Exploring the intersection of European law and national Criminal Law, ELReview 2000, 383-384, Frowein/Kirsch, der Rechtsschutz gegen Europol, JZ 1998, 589, Gleß, Europol, NStZ 2001, 623-624. Mit den Änderungen des Europolvertrags und der Erweiterung der Zuständigkeiten von Europol wird in der Zukunft das Hauptgewicht ihrer Betätigung wahrscheinlich auf andere Gebiete verschoben, wie z. B. auf die Teilnahme von Europol an gemeinsamen Ermittlungsteams.

39 Siehe dazu insbesondere Frowein-Kirsch, JZ 1998, 589ff., Gleß, NStZ 2001, 624ff aber auch Kaiafa-Gbandi, To poiniko dikaio stin Europaiki Enosi, 26ff, 192ff.

40 Siehe das Protokoll über die Vorrechte und Immunitäten für Europol, die Mitglieder der Organe, die stellvertretenden Direktoren und die Bediensteten von Europol, C 221 vom 19.7.1997, S. 2.

41 Siehe dazu Art. 2 des Protokolls beim Rechtsakt des Rates vom 28.11.2002, C 312 vom 16.12.2002, S. 3 und Ellermann, Vom Sammler zum Jäger-Europol auf dem Weg zu einem »europäischen FBI?«, ZeuS 2002, 580-582.

42 Siehe Art. 12 und 13 des Protokolls über die Vorrechte und Immunitäten für Europol. 
eigentlich nicht unabhängig ist. ${ }^{43}$ Somit weist die polizeiliche Zusammenarbeit im Rahmen der EU durch Europol einen Vorsprung nicht nur zeitlicher sondern auch qualitativer Art auf. Der wichtigste Grund dafür sind die bedeutenden Hindernisse für die justizförmige Kontrolle ihrer Tätigkeit. ${ }^{44}$

Für die Überwindung dieser wichtigen Defizite wurde angebracht gehalten, als Gegenwicht ein justizielles Organ für die Koordinierung der Ermittlungen und der Klageerhebungen im Rahmen der Union zu schaffen, auf dem verschiedene Hoffnungen gelegt wurden. ${ }^{45}$ Dennoch hat die Errichtung von Eurojust mit der konkreten Form, die sie genommen hat, diese Hoffnungen nicht erfüllt. ${ }^{46} \mathrm{Um}$ diese Stellungnahme klar zu machen erwähne ich paradigmatisch zwei Merkmale ihres institutionellen Aufbaus. Der erste betrifft ihre Zusammensetzung, die nicht nur aus Richtern oder Staatsanwälten aber auch aus Polizeioffizieren besteht. ${ }^{47}$ Eine solche Zusammensetzung, abgesehen von den Gründen ihres Vorzugs, führt notwendigerweise zur Entartung des Begriffs eines justiziellen Organs. ${ }^{48}$ Der zweite Punkt betrifft die Regelung der Beziehungen zwischen Europol und Eurojust. Obwohl Eurojust im Zuständigkeitsbereich von Europol aktiv wird, ${ }^{49}$ übt sie keine justizielle Kontrolle über Europol aus und dies ist klar in der Art und Weise, in der die Beziehungen dieser beiden Organe im Eurojust- Gründungsakt geregelt wurden..$^{50}$ Das war einerseits zu erwarten wegen des Mangels an strafrechtlicher Zuständigkeit der Union. Dennoch die vorgesehene, ohne wesentliche Abgrenzung, Zusammenarbeit von Eurojust mit Europol ist nicht problemlos. Und dies weil solange Europols rechtlicher Funktionsrahmen sich nicht ändert, die Defizite des Grundrechtsschutzes, die oben angesprochen wurden, notwendigerweise mit dem Informationsaustausch zwischen Europol und Eurojust auch auf dem Gebiet der Arbeit der Letzteren weitergeführt werden. Die Daten nämlich, die zu Eurojust von Europol weitergegeben werden, sind weiterhin von ihrer Immunitätsregelungen gedeckt. Somit übt Eurojust nicht nur keine Kontrolle über Europol sondern übernimmt eigentlich die von ihr eventuell rechtswidrig erworbenen oder bearbeiteten Daten, um in dieser Art und Weise weiterhin die Verbesserung der Zusammenarbeit zwischen den Mitgliedstaaten zu unterstützen. Mit anderen Worten, solange der rechtliche Rahmen von Europol sich diesbezüglich nicht ändert, hat die enge Zusammenarbeit mit Eurojust jede Möglichkeit als »guter Leiter«

43 Siehe insbesondere Frowein-Kirsch, JZ 1998, 592ff. und die Art. 24 Abs. 3 des Übereinkommens über die Errichtung von Europol.

44 von Bubnoff, ZeuS 2002, 195, Gleß, NStZ 2001, 624ff., Kaiafa-Gbandi, To poiniko dikaio stin Europaiki Enosi, 195.

45 Siehe Fn. 38

46 Siehe ausführlich Kaiafa-Gbandi, To poiniko dikaio stin Europaiki Enosi, 196ff.

47 Siehe Art. 2 des Ratsbeschlusses vom 28.2.2002 über die Errichtung von Eurojust, L 63 vom 6.3.2002, S. 2.

48 Vgl. Paraskevopoulos, I epidrassi tis synchronis anteglimatikis politikis sto poiniko dikonomiko dikaio, Poinika Chronika 2002, 587.

49 Siehe Fn. 48.

50 Art. 26 des Ratsbeschlusses vom 28.2.2002 (L 63 vom 6.3.2002, S. 9) spricht von »enger Zusammenarbeit« der beiden Organe. 
der Überführung von Defiziten des Grundrechtsschutzes auf dem Gebiet von Eurojust auch zu funktionieren. ${ }^{51}$

Dieses Ergebnis war voraussehbar und hat damit zu tun, dass man ohne die schon festgestellten rechtstaatlichen Defizite bei der Funktion verschiedener Organe der Union, die auf dem Gebiet der Kriminalitätsbekämpfung schon tätig waren, genügend in Betracht zu ziehen, neue Organe geschaffen hat und sie mit den älteren ohne weiteres in Formen enger Zusammenarbeit verbunden hat. Oder noch allgemeiner ausgedrückt: Das wesentliche Problem, das sich mit der Funktion von supra- oder transnationalen Organen im Rahmen der strafrechtlichen Verfolgung stellt, ist schließlich ein Problem des effektiven Grundrechtsschutzes. Solange dieser Schutz selbst nicht supranational organisiert wird, kann er nicht effektiv werden. Das ist zumindest bis heute bewiesen mit der Auswahl eines Models vom »Zweipolenschutz«, der in diesen Fällen sich zwischen den nationalen Gerichten und einer Gemeinsamen Kontrollinstanz bewegt. Der Versuch des Bürgers seine Rechte sicherzustellen, indem er sich zwischen diesen beiden Polen bewegt, garantiert den Erfolg nicht. Denn nicht nur der Weg des nationalen Schutzes ist eventuell mit Hindernissen behaftet -und charakteristisches Beispiel dafür sind die Europolimmunitäten- sondern auch die vorgesehenen Gemeinsamen Kontrollinstanzen werden von institutionellen Regelungsrahmen organisiert, die auch selbst Probleme eines defizitären Schutzes schaffen. $^{52}$

3. Das dritte und letzte Gebiet, auf dem ich den Abbau des Grundrechtsschutzes in der EU zeigen will, ist dieses ihres ersten Auftritts im internationalen Raum in Fragen der justiziellen Zusammenarbeit in Strafsachen mit der Unterzeichnung der Abkommen über die Auslieferung und die Rechtshilfe mit USA. ${ }^{53}$ Dieses Gebiet ist meines Erachtens wichtig für die Fragen, die uns hier beschäftigen, aus folgenden Gründen: Erstens, weil es die Regelung der Beziehungen der EU mit Drittstaaten betrifft und dadurch als Bezugspunkt die Außenbeziehungen der EU hat. Zweitens, weil es das erste Mal ist, wo die EU als eine Staatenorganisation Abkommen strafrechtlichen Charakters unterzeichnet und das macht evident, dass diese Abkommen als Prototyp wirken werden, ${ }^{54}$ sowohl bezüglich des Verfahrens wie auch ihres Inhalts für die Zukunft. Und drittens, weil der Staat mit dem diese Abkommen unterzeichnet wurden USA ist, also die einzige Supermacht in der Welt heute, die eine ganz bestimmte und wohl bekannte Politik in Fragen von Terrorismus und organisierter Kriminalität betreibt. ${ }^{55}$

51 Kaiafa-Gbandi, To poiniko dikaio stin Europaiki Enosi, 207-208.

52 O.a., 231-214.

53 Siehe den Ratsbeschluss vom 6.6.2003 (2003/516/EG), L 181 vom 19.7.2003, S. 25, das Abkommen zwischen der EU und den Vereinigten Staaten von Amerika über Auslieferung L 181 vom 19.7.2003, S. 27ff. und das Abkommen zwischen der EU und den Vereinigten Staaten von Amerika über Rechtshilfe L 181 vom 19.7.2003, S 34ff.

54 Siehe auch die relevante Schätzung des Europäischen Parlaments in P5_TAPROV(2003)0239.

55 Siehe insbesondere die Rechtsakte: »Patriot Act«, »Homeland security act« und »President's executive order on military tribunals $\ll$. 
Ich werde mich hier mit dem Unterzeichnungsverfahren der Abkommen nicht befassen, ${ }^{56}$ trotz der Tatsache, dass es sehr wichtige Fragen gestellt hat, die in engem Zusammenhang mit dem Grundrechtsschutz stehen. Ich meine damit die Verdrängung des Europäischen Parlaments wie auch die Tatsache der Behandlung der Abkommenentwürfe als geheim während der ganzen Zeit der Verhandlungen und die Aufhebung dieses Geheimstatus erst einen Monat vor der Ermächtigung der griechischen Präsidentschaft für deren Unterzeichnung im Juni 2003. Es soll hier nur klar sein, dass die Unterzeichnung dieser Abkommen von ihrem Abschluss gefolgt wird. Obwohl dieser vom Rat auch unternommen wird, setzt jedenfalls voraus, dass vorerst eventuelle verfassungsrechtliche Vorbehalte (Art. 24 Abs. 5 EUV) der Mitgliedsstaaten aufgehoben werden, die natürlich vorhin die entsprechende Zustimmung ihrer Parlamente sicherstellen sollen. ${ }^{57}$ Erfreulicherweise haben im Rat 12 Mitgliedstaaten solche Vorbehaltserklärungen geäußert, unter denen auch Deutschland, leider aber nicht Griechenland. ${ }^{58}$

Das was mich hier beschäftigen wird, sind manche Regelungen dieser Abkommen, die meiner Meinung nach den Abbauprozess der Grundrechte zeigen können. Damit mein Versuch verständlich wird, sollte im voraus gesagt werden, dass das Hauptmerkmal der obengenannten Abkommen ihre grundsätzlich ergänzende Funktion zu den schon geltenden bilateralen Verträgen zwischen den jeweiligen Mitgliedstaaten und USA ist. Mit anderen Worten, mit diesen Abkommen wird für jeden Mitgliedstaat ein neuer Rahmen für die Auslieferung und die Rechtshilfe mit USA geschaffen, der sich von den eventuell schon geltenden bilateralen Verträgen und deren »Verbesserung « mit den neuen Regelungen, die die EU für alle Mitgliedstaaten unterschrieben hat, bestehen wird. Dies erklärt auch, warum der Rat in Bezug auf diese Abkommen betont, dass sie einen »Mehrwert « $\mathrm{zu}$ den schon existierenden bilateralen Verträgen der Mitgliedstaaten mit USA anbieten. Eine Schätzung natürlich, die ihrem Wesen nach untersuchungsbedürftig ist.

Im Auslieferungsabkommen soll man unbedingt zwei Regelungen in Betracht ziehen. Die erste betrifft die Auslieferung in Fällen, wo die Straftat, wegen der um Auslieferung ersucht wird, nach den Gesetzen des ersuchenden Staates mit der Todesstrafe bedroht wird. Dieses Thema wird im Artikel 13 des Abkommens geregelt, der vorsieht, dass der ersuchte Staat die Auslieferung gewähren kann, unter der Bedingung, dass die Todesstrafe nicht verhängt wird, oder -wenn eine derartige Bedingung vom ersuchenden Staat aus Verfahrengründen nicht erfüllt werden kann- unter der Voraussetzung, dass die Todesstrafe, falls verhängt, nicht vollstreckt wird. Wenn der ersuchende Staat die Auslieferung unter diesen Bedingungen akzeptiert, hat er sie zu erfüllen, anders wird der Auslieferungsantrag nicht obligatorisch abgelehnt, sondern er darf einfach abgelehnt werden. Diese Regelung schafft ein großes Problem sowohl für

56 Siehe dazu ausführlich Kaiafa-Gbandi, To poiniko dikaio stin Europaiki Enosi, $241 \mathrm{ff}$.

57 Über das Verfahren vom Art. 24 EUV für solche Verträge siehe Calliess/Ruffert, Kommentar zu EU-Vertrag und EG-Vertrag, 2. Auflg, 2002, 209ff und bezüglich der Abkommen mit USA Papadopoulou, I didymes symfonies ekdosis kai amiveas dikastikis synergasias EE-IPA, Nomiki vasi, euros tis enosiakis armodiotitas kai diadikasia synomologisis, ToS, $3 / 2003$.

58 Siehe das Ratsdokument 10409/2003 vom 13.6.2003. 
die EU wie auch für die Mitgliedstaaten, die die Todesstrafe abgeschafft haben, insbesondere wenn dies aufgrund ihrer Verfassungen oder der Ratifizierung von internationalen Verträgen mit übergesetzlicher Kraft passiert ist.

Die EU selbst ist nämlich aufgrund vom Art. 6 Absatz 2 des EUV an die Achtung der Grundrechte gebunden, wie sie in der EMRK gewährleistet werden. Das 6. Protokoll von EMRK hat die Todesstrafe abgeschafft und erlaubt nicht mal die Bedrohung ihrer Auferlegung, mit einziger Ausnahme der Kriegsverbrechen. Andererseits schafft das 13. Protokoll der EMRK, welches im Sommer 2003 auch in Kraft getreten ist, die Todesstrafe unter allen Umstände ab, wobei die Charta der Grundrechte der Union im Art. 19 ein Verbot der Auslieferung im Falle des Bestehens eines ernsthaften Risikos der Todesstrafe ausdrücklich vorsieht. Trotz der obengenannten Bindungen hat die EU ein Abkommen unterzeichnet, nach dem in solchen Fällen eine ad hoc Verständigung stattfinden soll und auf der Basis einer Verhandlung offensichtlich versucht wird, eine amerikanische Justizbehörde zu binden, die Todesstrafe nicht aufzuerlegen oder im Falle ihrer Auferlegung sie nicht zu vollstrecken. Dieses Schema setzt vor allem die gesuchte Person der Unsicherheit der Verhandlungen aus und insbesondere der tatsächlichen Einhaltung ähnlicher ad hoc Bindungen.

Gleich problematisch -und zwar im Rahmen der gleichen Philosophie der Umwandlung des Grundrechtsschutzes zum Verhandlungsobjekt- erscheinen im Auslieferungsabkommen auch die Regelungen über den Schutz der persönlichen Daten und der vorübergehenden Überstellung der gesuchten Person, die im ersuchten Staat eine Strafe verbüßt oder gegen die ein Verfahren anhängig ist. ${ }^{59}$

Besonders wichtig im Auslieferungsabkommen ist andererseits die Rolle von Art. 17, dessen 2. Absatz Konsultationen unter den betroffenen Staaten vorsieht, wenn die Verfassungsgrundsätze des ersuchten Staates oder die für ihn verbindlichen endgültigen richterlichen Entscheidungen ein Hindernis für die Erfüllung seiner Auslieferungspflicht darstellen und dieses Abkommen oder der geltende bilaterale Vertrag keine Regelung für diese Angelegenheit vorsehen. Es wird darauf hingewiesen, dass diese Regelung darauf zielte, dass es den Mitgliedstaaten ermöglicht, weiterhin den Auslieferungsantrag aufgrund ihrer verfassungsrechtlichen Prinzipien abzulehnen. Man kann aber nicht übersehen, dass die Formulierung des Abkommens selbst vielmehr die Auffassung unterstützt, dass sogar die verfassungsrechtlichen Hindernisse mit Konsultationen zu überwinden seien, ${ }^{60}$ wobei es, wenn solche Hindernisse tatsächlich existieren, evident ist, dass über die verfassungsrechtliche Bindung keine Konsultationen geben kann.

59 Artikel 14 und 9 Abs. 2 entsprechend.

60 Siehe dazu auch kritisch Beis, Ekdosi stis IPA kai ellimma tou kratous dikaiou, Efimerida »Eleutherotypia« vom 25. 6.2003, S. 9, Mangakis, Mia antitromokratiki symvasi pou tromazi, Efimerida Eleutherotypia, 10.6.2002, Spiliotopoulou/Koukouli-Spiliotopoulou, Symfonies ekdosis kai dikastikis syndromis metaxi EE kai IPA-Paratirisis schetika me ti synapsi kai tin ischi tous, in Idryma Maragopoulou gia ta Dikaiomata tou Anthropou, I symfonies ekdosis kai dikastikis syndromis EE-IPA: Provlimata gia ta dikaiomata tou Anthropou, Athina 2003, 6-7. 
Ähnliche Defizite findet man auch beim Abkommen über die Rechtshilfe. ${ }^{61}$ Diese konzentrieren sich hauptsächlich auf die Regelungen über die gemeinsamen Ermittlungsteams und die Begrenzungen bei der Verwendung von personenbezogener Daten. Was die gemeinsamen Ermittlungsteams anbelangt, sieht das Abkommen eine offene Zweckmäßigkeitsentscheidung über ihre Bildung vor, die offensichtlich die USA als Supermacht begünstigt, während die Funktionsregeln solcher Ermittlungsteams einer ad hoc Entscheidung der zuständigen Behörden der betreffenden Staaten allein zustehen(!). ${ }^{62}$ Andererseits durch eine erläuternde Note des Abkommens zu Art. 9, welches die Verwendung personenbezogener Daten betrifft, wird klar, dass » für die Ablehnung der Rechtshilfe Datenschutzgründe nur in Ausnahmenfälle geltend gemacht werden können «, aber auch dass »die kategorische oder systematische Ablehnung der Zusammenarbeit aufgrund einer extensiven Auslegung der Datenschutzgrundsätze auszuschließen ist $\ll .{ }^{63}$ Mit einem solchen Regelungsrahmen fragt man sich, ob der Analogiegrundsatz oder vielmehr der institutionelle Schutz der persönlichen Daten in einem Staat noch einen Wert hat. ${ }^{64}$ Denn der Letztere wird wohl vorgesehen gerade für den allgemeinen und systematischen Schutz der Rechte der Bürger und nicht als eine Ausnahmefallregelung.

Anhand der vorgeführten Bemerkungen wird ersichtlich, dass die Abkommen mit USA als Hauptzweck die Effektivität der Kriminalitätsbekämpfung hatten und für diesen Zweck den Grundrechtsschutz von seiner Basis her in Frage stellen. Sie führen eine ganz neue Philosophie des Grundrechtsschutzverfahrens ein und sogar dort auch, wo es verfassungsrechtliche Regelungen oder internationale Verträge gibt, die ihn in seiner heutigen Form gewährleisten. Der Schutz der Grundrechte ist nunmehr möglich zum Verhandlungsobjekt der Parteien zu werden, damit »die Hindernisse « aufgehoben werden, die sein institutioneller Rahmen bereitet. Somit werden sie im »Marktsystem « eingeführt. Deren Schutz gibt es nämlich je nach dem Maß, den das Angebot und die Nachfrage für ihn mitbestimmen. Ich glaube nicht, dass man mehr dazu sagen sollte, um diese negative »Errungenschaft« der EU am Anfang des 21. Jahrhunderts zu charakterisieren. Wichtig ist nur vor Augen zu halten, dass die EU hier über den Grenzen ihrer rechtlich bindenden Kompetenz gehandelt hat, denn Art. 6 Abs. 2 EUV bindet sie an die Achtung der Grundrechte so wie von der EMRK gewährleistet werden.

Wenn man die obengenannten drei Beispiele vom Abbau des Grundrechtsschutzes vergleicht, stellt man fest, dass im Innenraum der EU ein Abbauprozess stattfindet, der entweder auf der Ebene der Anwendung von Strafrechtsprinzipien oder auf dieser der Schutzmechanismen der Bürger die Grenzen eines geltenden, wenn auch defizitären, institutionellen Grundrechtsschutzrahmens auf die Probe stellt. Im Gegensatz dazu, sobald man sich zu der Ebene der Außenbeziehungen der EU mit Drittstaaten bewegt, darf der institutionelle Schutzrahmen sogar beiseite gelassen werden. Der Abbaupro-

61 Dazu auch ausführlicher Kaiafa-Gbandi, To poiniko dikaio stin Europaiki Enosi, 264ff.

62 Kritisch auch Paraskevopoulos, Symvasi dikastikis syndromis EE-IPA, Efimerida »Eleutherotypia«, 2.7.2003, 9. Vgl. auch die ausführlichen Regelungen des Rahmenbeschlusses über die gemeinsamen Ermittlungsteams in der EU, L 162 vom 20.6.2002, S. 1-3.

63 L 181 vom 19.7.2003, S. 41.

64 Siehe auch Paraskevopoulos, o. a. 
zess der Grundrechte erreicht somit seinen Höhenpunkt und der Bürger kann nicht mal im voraus wissen, worauf er sich stützen soll, da die wichtigsten Punkte im Bezug auf seinen Schutz je nach Fall und mit ad hoc Vereinbarungen festgelegt werden.

Das Zurückgehen der staatlichen Souveränität gegenüber supranationalen Organisationen, wie die EU, funktioniert, wie man sieht, in einer multiplizierenden Art für den Abbau der Grundrechte. D.h. obwohl die EU mit ihren Organen neue Angriffschancen für die Grundrechte schafft, kontrolliert sie diese Angriffe auf supranationaler Ebene nicht, weder unterwirft sie sich selbst als selbständiger Machtträger zu Kontrollschemata des Grundrechtsschutzes, wie es die jeweiligen Staaten tun. ${ }^{65}$ Außerdem ist sie anscheinend noch der Meinung, dass bei ihren Beziehungen mit Drittstaaten als neuer Machtträger das Grundrechtsschutzsystem von neu an wiederverhandeln kann. Somit wird jede Errungenschaft, die auf europäischer Ebene für den Grundrechtsschutz erlangt wurde, wieder in Diskussion gestellt und zwar nicht in dem Sinn einer Neufestlegung von institutionellen Schutzmindestgrenzen.

\section{Die Perspektiven nach dem Verfassungsentwurf für Europa}

Die nächste Frage, die sich stellt, ist natürlich, inwieweit die Situation sich verbessern würde durch die angestrebten Veränderungen in der EU auf der Basis des Entwurfs einer Verfassung für Europa, dessen Unterstützung, trotz des Misserfolgs der intergouvernementalen Konferenz in Rom im Dezember 2003, nicht aufgegeben worden ist.

Die Antwort soll hier mit Vorsicht formuliert werden, denn manche wichtigen Punkte sind immer noch nicht genügend geklärt. Dennoch anhand dessen, was man aus dem Entwurf selbst erlesen kann, würde ich sagen, dass die geplanten Verbesserungen nicht ausreichen, damit man auf eine wesentliche Wende der Entwicklung des Strafrechts in der EU und somit des Grundrechtsschutzes hoffen kann.

Diese Stellungnahme basiert sich erstens darauf, dass die Sicherheit weiterhin das Grundziel der EU im Rahmen der Strafsachen darstellt und zwar in ihrem Verfassungsentwurf in noch aufgehobener Weise, wie man vom Art. III-158 feststellen kann. ${ }^{66}$

65 Das erklärt auch den Unterschied zum Abbau des Grundrechtsschutzes, den man auf nationaler Ebene auch im Bereich eines Sicherheitsstrafrechts feststellen kann. Deshalb erscheint dieser Prozess im Rahmen der EU noch gefährlicher. Trotzdem ist es evident, dass eine erfolgsversprechende Behandlung des Problems nur dann möglich wäre, wenn man den Abbau des Grundrechtsschutzes im Bereich des Strafrechts auf beiden Ebenen, also sowohl national wie auch europäisch, anzuhalten versuchen würde.

66 Art. III-158 ist dem Raum der Freiheit, der Sicherheit und des Rechts gewidmet. Obwohl darin im ersten Absatz angegeben wird, dass in diesem Raum die Grundrechte geachtet werden, ist in den folgenden drei Absätze leicht feststellbar, dass »die Freiheit « ausschließlich in Verbindung mit der freien Bewegung in den Innengrenzen der EU betrachtet wird, »das Recht« mit der Erleichterung des Zugangs dazu und insbesondere mit der gegenseitigen Anerkennung gerichtlicher Entscheidungen, wobei die hauptsächlichen Bemühungen der Union sich auf die Gewährleistung »der Sicherheit « konzentrieren, da sie das einzige der drei vorerwähnten Elemente ist, wofür »ein hohes Niveau« erstebt wird. Dennoch wenn die Sicherheit nicht in einer parallelen und ausgeglichenen Beziehung mit der Freiheit der Bürger erstrebt wird, die natürlich aus vielen anderen Elementen auch als die freie Bewe- 
Andererseits sind Probleme auch außerhalb der allgemeinen Verkündungen leicht feststellbar. Die Intervention der EU bei der Festlegung von Straftaten in den sie interessierenden Gebieten wird z.B. nach Art. III-172 des Entwurfs in Form des Erlasses von europäischen Rahmengesetzen vorgesehen, die die Mindestvorschriften festlegen und die Mitgliedsstaaten dadurch im Bezug auf dessen, was zumindest strafbar gehalten wird und mit welcher Mindest-Höchstgrenze von Strafe bedroht wird, binden. Dennoch mag das demokratische Defizit beim Erlass solcher Rahmengesetze mit dem Mitbestimmungsrecht des Europäischen Parlaments verringert sein, es wird jedoch nicht eliminiert. ${ }^{67}$ Und dies ist wichtig bei der Strafbarkeitsbestimmung, nicht nur weil der n.c.n.p.s.l.-Grundsatz nur als Ausdruck des Demokratieprinzips einen Sinn hat, sondern auch weil dieses bedeutende Defizit der Rahmengesetze, als bindende Regel für ein Mindestergebnis, mit der weiteren Intervention der nationalen Parlamente nicht geheilt werden kann. ${ }^{68}$ Wenn man sogar denkt, dass nach dem Verfassungsentwurf die Mitgliedstaaten für den Verstoß gegen ihre Verpflichtung, ein europäisches Rahmengesetz in die nationale Rechtsordnung umzusetzen, viel effektiver kontrolliert werden können, d. h. durch die Zahlung eines Pauschalbetrags oder eines Zwangsgelds, ${ }^{69}$ dann wird es ersichtlich, dass die EU in der Zukunft in der Lage sein wird, ihre Sicherheitspolitik in den Mitgliedstaaten viel dynamischer durchzusetzen.

Andererseits erscheint die Lage auf der Ebene der Strafbarkeitsfestlegung speziell für den Betrug gegen die finanziellen Interessen der Union noch problematischer zu sein, denn die entsprechenden Delikte werden im Fall der Errichtung einer Europäischen Staatsanwaltschaft in europäischen Gesetze festgelegt, also ohne jede Intervention der nationalen Parlamente und zwar in Gesetzen des Ministerrates, für die einfach die Zustimmung des Europäischen Parlaments erforderlich ist (Art. III-175 Abs. 2),

gung in den Innengrenzen besteht, dann ist es evident, dass diese Sicherheit mit einem Rechtsstaat nichts zu tun hat. Vgl. dazu den Änderungsvorschlag für Art. III-158VE beim Alternativ-Entwurf »Europäische Strafverfolgung «, der bei der dt. Strafrechtlertagung vom 7/8.11.2003 in Dresden vorgestellt wurde.

67 Anders A. Psarouda-Benakis, Pros ena »Europaiko« Poiniko Dikaio?, Poinika Chronika 2003, 967. Für die Regelung des Verfassungsentwurfs siehe Art. 33 Abs.1 und III-302 VE und vgl. auch den Vorschlag für eine Änderung vom Art. III-302 beim Alternativ-Entwurf »Europäische Strafverfolgung « (dt. Strafrechtlertagung, Dresden 2003, S. 18-21). Nach diesem Änderungsvorschlag heißt es (Art. III-302 Abs. 3) bei der ersten Lesung: » Handelt es sich um ein Strafgesetz, so erfolgt eine Übermittlung an den Ministerrat nur, wenn sich 2/3 der Mitglieder des EU-Parlaments für den Erlass des Gesetzes aussprechen«. Der Vorschlag für die 2/3 Mehrheit wird dadurch begründet, dass bei der Wahl zum EU-Parlament der Grundsatz der Wahlrechtsgleichheit noch nicht gewahrt ist und die nach dem VE gegebene Möglichkeit Strafgesetze gegen die Repräsentanten der Mehrheit der Bevölkerung zu verabschieden, mit dem demokratischen Gedanken nicht zu vereinbaren ist.

68 Vgl. auch Psarouda-Benakis, Poinika Chronika 2003, 968-969.

69 Siehe Art. III-267 Abs. 3 des VE und Skouris, I protasis metarythmisis tou koinotikou systimatos dikastikis prostasias me aformi to schedio Syntagmatos tis Europaikis Enosis, in Tomeas Dimosiou Dikaiou kai Politikis Epistimis, Tmima Nomikis A.P.Th., Athina-Thessaloniki, 2003, 343ff. Skouris macht darauf aufmerksam, daß wenn schon die Zahlung eines Zwangsgelds für die Nicht-Mitteilung der Umsetzung eines europäischen Rahmengesetzes verhängt wird, desto mehr sollte das für die Nicht-Umsetzung des Rahmengesetzes selbst gelten. 
d. h. mit europäischen Gesetzen, die nicht dem ordentlichen Gesetzgebungsverfahren vom Art. III-302 des Verfassungsentwurfs folgen, mit dem Ergebnis, hier wiederum eine Vergrößerung des Demokratiedefizits feststellen zu können.

Anhand solcher Bestimmungen fürchte ich, dass keine wesentliche Sicherstellung des Bürgers gegen eine übermäßige Erweiterung der Strafbarkeit erreicht wird. Im Verfassungsentwurf für Europa ist es sogar offensichtlich, dass das Hauptziel für die Union vielmehr die Effektivität bei der Kriminalitätsbekämpfung als eine Harmonisierung wichtiger Delikte durch den Beitrag der verschiedenen Mitgliedstaatstraditionen ist. Den schnellen und einfachen Weg dafür stellt das Prinzip der gegenseitigen Anerkennung der gerichtlichen Entscheidungen mit Überwindung des »irritierenden « Prinzips der doppelten Strafbarkeit dar, obwohl das zu einem gefährlichen Rückgang der Bürgerrechte und zu einer europaweiten Exekutierbarkeit der punitivsten Strafrechtsordnung führen kann. ${ }^{70}$

Eine Verbesserung der Lage für die Grundrechte kann meiner Meinung nach auch nicht beim institutionellen Regelungsrahmen der Unionsorgane im Bereich der polizeilichen und justiziellen Zusammenarbeit festgestellt werden. Das kann man charakteristisch durch Art. III-175, ${ }^{71}$ III-176 Abs.3 und III-178 des Entwurfs feststellen, die in besonders wichtigen Fällen, wie die Errichtung einer Europäischen Staatsanwaltschaft, die Festlegung von gemeinsamen operativen Maßnahmen der polizeilichen Behörden der Mitgliedstaaten, wie auch die Festlegung der Bedingungen, unter denen die zuständigen Behörden eines Mitgliedstaats im Hoheitsgebiet eines anderen operieren können, als Rechtsinstrumente europäische Gesetze oder Rahmengesetze des Ministerrates mit einfacher Anhörung oder Zustimmung des Europäischen Parlaments vorsehen. Man merkt dadurch, dass je wichtiger das Interventionsgebiet für die Union desto rückgängiger das Demokratieprinzip wird.

Das Bild kann wohl nicht vollkommen sein, wenn man hier nicht parallel den Verfassungsentwurf im Bezug auf dessen Teil untersucht, welcher dem Grundrechtsschutz selbstständig gewidmet wird. Die Anerkennung bindender Kraft zu den Grundrechten mit ihrer Eingliederung im zweiten Teil des Verfassungsentwurfs stellt sicherlich einen wichtigen Fortschritt. Die nun vorgesehene institutionelle Bindung der Unionsorgane an rechtsstaatliches Handeln ist sicherlich bedeutend. Dennoch hängt das Niveau des angebotenen Schutzes, wie bekannt, entscheidend vom Schutzmechanismus ab, der dafür bereit gestellt wird.

Als erstes ist diesbezüglich wichtig zu betonen, dass die EU mit dem Verfassungsentwurf EMRK nicht beitritt, sondern sie erklärt einfach, dass sie diesen Beitritt weiter

70 Art. III- 158 Abs. 3 und III-171 Abs. 1 VE. Kritisch zu einem solchen Prinzip Kaiafa-Gbandi, To poiniko dikaio stin Europaiki Enosi, 37ff, Schünemann-Nestler, Thesen zur »Europäisierung der Strafverfolgung durch das Prinzip der gegenseitigen Anerkennung «, Alternativ-Entwurf »Europäische Strafverfolgung «, dt. Strafrechtlertagung, Dresden, 2003, S. 48-49.

71 Vgl. dazu auch den Änderungsvorschlag vom Alternativ-Entwurf »Europäische Strafverfolgung «, dt. Strafrechtlertagung, Dresden, 2003, S. 14-17. 
anstrebt. $^{72}$ Die Grundrechte, wie sie von der EMRK gewährleistet werden, gehören also weiterhin zu den allgemeinen Grundsätze des Unionsrechts ${ }^{73}$ und somit ändert sich das Verhältnis der EU zur EMRK auch mit dem neuen Schritt des Verfassungsentwurfs nicht.

Wenn man nun das Gebiet des gerichtlichen Schutzes, der anerkannten Grundrechte in Betracht zieht, stellt man fest, dass obwohl Art. III-270 Abs. 4 des Verfassungsentwurfs den rechtlichen Schutz der Bürger nach der Eingliederung der Grundrechtscharta in der Verfassung erweitert hat, macht er trotzdem aufgrund seiner Formulierung nicht klar, ob die Individualbeschwerde, die für natürliche und juristische Personen anerkannt wird, außer den Fällen von Organhandlungen, die an die Individuen ergangen sind oder sie unmittelbar und individuell betreffen und aus Rechtsmäßigkeitsgründen angefochten werden können, auch alle andere Gesetzgebungsakte sowie Rechtsakte ohne Gesetzescharakter nach der Unterscheidung von Art. 32 bis 34 betrifft, im Falle dass solche Akte ihre Ergebnisse unmittelbar bringen und keine Durchführungsmaßnahmen brauchen. ${ }^{74}$ Als vorzuziehende wird diesbezüglich die engere Lösung vorgeschlagen, die nämlich die europäischen Gesetze und Rahmengesetze mit einer Individualbeschwerde als nicht anfechtbar hält. ${ }^{75}$ Sollte eine solche Auslegung sich durchsetzen, dann ist es klar, gerade weil die polizeiliche und justizielle Zusammenarbeit in Strafsachen von der Union ausschließlich mit europäischen Gesetzen und Rahmengesetzen geregelt wird, dass der Schutz der Bürger auf diesem Gebiet beschränkt sein wird.

Aber auch wenn man der Individualbeschwerde die weitest mögliche Funktion im Rahmen von Art. III- 270 Abs. 4 einräumen würde und der Verfassungsentwurf tatsächlich in Kraft treten würde, bestünde, meiner Meinung nach, weiterhin ein wichtiges Problem. Das hat nicht so sehr mit dem Auswahlverfahren der Richter zu tun, die nach Art. III-260 Satz a von den Regierungen ernannt werden, ${ }^{76}$ sondern hauptsächlich mit der Tatsache, dass der Gerichtshof der EU als Bestandteil ihres institutionellen Systems auch in der Vergangenheit gezeigt hat, dass er bei seinen Urteilen die Unterstützung der Unionsziele der Rechtseinhaltung selbst nicht selten vorzieht. Man könnte hier als Beispiel an seine Stellungnahme denken, über den Charakter der besonders hohen Geldbußen, die von der Union auferlegt werden und die als Verwaltungssanktionen und nicht als Strafen im Gegensatz zu der entsprechenden Rechtsprechung des Europäischen Gerichtshofs für Menschenrechte gehalten werden. ${ }^{77}$

$72 \mathrm{Zu}$ den Schwierigkeiten dieses Beitritts siehe Kousis, I prostasia ton themeliodon dikaiomaton stin Europaiki Enosi, EEEurDik 2003,608-609 und Papadopoulou, O Europaikos Chartis kai I prostasia ton themeliodon dikaiomaton stin enosiaki ennomi taxi, To Syntagma 2001, 1284-1286.

73 Art. 7 VE.

74 Siehe Skouris, I protasis metarythmisis tou koinotikou systimatos dikastikis prostasias me aformi to schedio Syntagmatos tis Europaikis Enosis, o.a., $346 \mathrm{ff}$.

75 So Skouris, o.a., 349-350.

76 Siehe aber Skouris, o.a., 339-340, der das nicht für problematisch hält.

77 Mylonopoulos, Ta dogmatika themelia tou poinikou dikaiou stin nea chilietia, in Enosi Ellinon Poinikologon, To poiniko dikaio sto neo diethnes perivallon, Athina 2001, 49-51. 
Mit anderen Worten meine ich, dass welche Verbesserung auch immer sich mit der Anerkennung der Grundrechte des Bürgers im Verfassungsentwurf und der entsprechenden Möglichkeit einer Individualbeschwerde für sie gegen Handlungen der Unionsorgane ereignet hat, kann nicht als ausreichend gehalten werden. Nicht nur weil sie insbesondere für das Gebiet der polizeilichen und justiziellen Zusammenarbeit in Strafsachen nicht breit genüg angelegt ist, sondern hauptsächlich weil man für den Grundrechtsschutz im Rahmen der Union einen eigenständigen Gerichtshof, unabhängig vom übrigen gerichtlichen Mechanismus der Union bräuchte, der sich nicht als Bestandteil ihres institutionellen Rahmens verstehen würde, sondern ausschließlich als Träger des Grundrechtsschutzes der Bürger. Meines Erachtens ist die Tatsache, dass der Europäische Gerichtshof für Menschenrechte einen besonders erfolgreichen Gang vorzuzeigen hat, dazu zu verdanken, dass er eine außenstaatliche Institution ist, die dem Grundrechtsschutz ausschließlich gewidmet wird. ${ }^{78}$ Deshalb stellt der Beitritt der Union zur EMRK eigentlich den wichtigsten Weg dar für eine wesentliche Verbesserung des Grundrechtsschutzes innerhalb ihres Rahmens. ${ }^{79}$ Diese Verbesserung ist heute aufgrund der massiven Aktivität der Union im Bereich der Strafsachen mehr als je erforderlich und trotz der existierenden Probleme beim Europäischen Gerichtshof für Menschenrechte ${ }^{80}$ wie ich meine, auch praktisch erreichbar.

\section{Die Herausforderung für die Strafrechtswissenschaft}

Die Entwicklung des Strafrechts und insbesondere des Europäischen Strafrechts in der heutigen Zeit lässt natürlich auch die Rolle der Strafrechtler nicht unangetastet. Im Gegensatz zu der letzten Hälfte des vorigen Jahrhunderts haben das wissenschaftliche Wort und die Vision für ein rechtsstaatliches Strafrecht keine gute Chancen für ihre Umsetzung in die Praxis. Schon die Internationalisierung des Strafrechts verringert eine solche Möglichkeit in bedeutender Weise. Gerade deshalb ist es aber, wie ich meine, unsere Verantwortung noch größer geworden. Wir sind dazu gerufen, mehr denn je der Bürgergesellschaft bei ihrem Versuch, sich einem globalisierten Sicherheitsrecht zu widersetzen, Hilfe zu leisten und dies kann nicht ohne einen effektiven Grundrechtsschutz stattfinden. Natürlich wird es auf diesem Weg gar nicht einfach

78 Vrgl. dazu Spinellis, Porismata apo ti nomologia tou Europaikou Dikastiriou ton Dikaiomaton tou Anthropou schetika me to poiniko dikaio, ousiastiko kai dikonomiko, in Ethniki Scholi Dikaston, I epidrasi tis Europaikis Symvasis ton Dikiomaton tou Anthropou stin erminia kai efarmogi tou ellinikou dikaiou, Athina-Komotini, 2002, 199-200.

79 Siehe diesbezüglich Frangaki, Anthropina dikaiomata kai europaiki enopoiissi, Nomiko Vima 2002, 1425. Vgl. auch Rozaki, O Chartis ton themeliodon dikaiomaton tis Europaikis Enosis, EEEurDik 2001 (eidiko teuchos), 439. Über den Beitritt der EU zur EMRK und die Sicherstellung der Grundrechte im Rahmen der Union als jedenfalls nicht gegeneinander wirkend oder vielmehr einander ausschließend siehe auch Papadopoulou, To Syntagma 2001, 1277 und 1882ff.

80 Siehe diesbezüglich Chrysogonos, I Europaiki Symvyasi ton Dikaiomaton tou Anthropou. Miso aiona meta, in Ethniki Scholi Dikaston, I epidrasi tis Europaikis Symvasis ton Dikaiomaton tou Anthropou stin erminia kai efarmogi toz ellinikou dikaiou, Athina-Komotini, 2002, 72-74. 
sein, über die Notwendigkeit eines solchen Vornehmens zu überzeugen, denn schon die Änderung in der Erfassung der Menschenrechte, als seitens Dritter und nicht des Staates angreifbarer und zu schützender Werte, begünstigt die einseitige Verbreitung der Strafbarkeit, mit der man meint, dass der Staat oder die internationalen und supranationalen Organisationen ihre Schutzaufgaben erfüllt hätten. ${ }^{81}$ Deshalb halte ich es für wichtig, dass wir zeigen, wie und in welcher Tiefe die Grundrechte heute abgebaut werden und welche die Wege für eine bessere Zukunft sind. Zu diesen Wegen ${ }^{82}$ gehört nicht nur der Justizmechanismus zum Schutz der Grundrechte, sondern beispielsweise auch eine Grundrechtsbeobachtungsstelle, ein für den Grundrechtsschutz zuständiger Kommissar, die systematische Dokumentierung von Grundrechtsverstöße im Rahmen der EU u.s.w. ${ }^{83}$ Außerdem sollte man längst neben dem europäischen Ombudsmann dem Europäischen Datenschutzbeauftragten Aufgaben für den Schutz der persönlichen Daten gegen die Eingriffe von Unionsorgane wie Europol und Eurojust anerkennen. ${ }^{84}$ Mit solchen Maßnahmen könnte man schon einiges auf einer präventiven Ebene verbessern. Dazu müsste auch eine bessere und engere Zusammenarbeit der nationalen Parlamente in Fragen der Aktivitäten der EU und insbesondere natürlich im Bereich der Strafsachen stattfinden. Hierzu sollte sich die schon bestehende Institution von $\operatorname{COSAC}^{85}$ viel aktiver einsetzen. Und »last but not least« kämen natürlich die Änderungen auf institutioneller Ebene, die ich schon vorhin angesprochen habe, d.h. bessere Sicherstellung der Grundrechte in der Verfassung für Europa insbesondere auf dem Gebiet der polizeilichen und justiziellen Zusammenarbeit in Strafsachen, Gewährleistung des Demokratieprinzips bei der legislativen Tätigkeit der Union in Strafsachen und Beitritt zur EMRK. Dadurch glaube ich, dass wir einen längst notwendigen Beitrag zu »unserer vergessenen europäischen Freiheit ${ }^{86}$ geleistet hätten, damit sie allmählich wieder in Erinnerung gerufen wird.

81 Siehe dazu die tiefgreifende Analyse von Günther, Diethnis anteglimatiki politiki: I poria pros ena pagosmio dikaio asfalias, (o.a.), 27-28. Um sich diese Entwicklung in der Erfassung der Grundrechte zu widersetzen ist wichtig zu zeigen, worin die falschen Ansätze einer solchen Auffassung liegen, welche ihre Folgen sind und insbesondere wie leicht jeder Bürger sich an der Stelle der »schwarzen Schaffe « befinden kann (vgl. Günther, o.a., 40ff.).

82 Siehe dazu auch Hassemer, Ein Strafrecht für Europa, in Zieschang/Hilgendorf/Laubenthal (Hrsg.), Strafrecht und Kriminalität in Europa, 21ff. und zu den theoretischen Grindlagen der zu verfolgenden Wege Prittwitz, ZStW 2001, 793ff.

83 Dazu Papadopoulou, To Syntagma 2001, 1276 mit weiteren bibliographischen Angaben.

84 Der Anwendungsgebiet der Verordnung 45/2001 EG, die die Institution des Europäischen Bürgerdatenschutzbeauftragten vorsieht, ist gemäß Art. 3 nur auf dem Bereich des Gemeinschaftsrechts beschränkt.

85 Es handelt sich um die Konferenz der Ausschüsse für Gemeinschafts- und Europa- Angelegenheiten der Parlamente der EU-Mitgliedstaaten. Für ihre Geschäftsordnung siehe C 235 vom 1.10.2003, S. 1-4.

86 Siehe dazu das Werk von P. A. Albrecht, Die vergessene Freiheit, Berlin 2003. 